\title{
Fundus optic disc localization and segmentation method based on phase congruency
}

\author{
Lei Geng ${ }^{\mathrm{a}}$, Yi-Ting Shao ${ }^{\mathrm{a}}$, Zhi-Tao Xiao ${ }^{\mathrm{a}, *}$, Fang Zhang ${ }^{\mathrm{a}}$, Jun $\mathrm{Wu}^{\mathrm{a}}$, Min $\mathrm{Li}^{\mathrm{a}}$ and Chun-Yan \\ Shan ${ }^{\mathrm{b}}$ \\ ${ }^{a}$ School of Electronics and Information Engineering, Tianjin Polytechnic University, Tianjin 300387, \\ China \\ ${ }^{b}$ Metabolic Disease Hospital, Tianjin Medical University, Tianjin 300070, China
}

\begin{abstract}
It has been demonstrated that shape, area and depth of the optic disc are relevant indices of diabetic retinopathy. In this paper, we present a new fundus optic disc localization and segmentation method based on phase congruency (PC). Firstly, in order to highlight the optic disc, channel images with the highest contrast between optic disc and background are selected in LAB, YUV, YIQ and HSV spaces respectively. Secondly, with the use of PC, features of four selected channel images can be extracted. Multiplication operation is then used to enhance PC detection results. Thirdly, window scanning and gray accumulating are utilized to locate the optic disc. Finally, iterative OTSU automatic threshold segmentation and Hough transform are performed on location images, before the final optic disc segmentation result can be obtained. The experimental results showed that the proposed method can effectively and accurately perform optic disc location and segmentation.
\end{abstract}

Keywords: Fundus image, phase congruency, optic disc localization, optic disc segmentation, diabetic retinopathy

\section{Introduction}

Rapid developing image processing technology is widely being applied in the field of medical research [1]. Diabetic retinopathy (DR) is one of the most serious complications of diabetes mellitus (DM). Optic disc is an important component in fundus image as its characteristics (such as shape and size) are auxiliary parameters in clinical diagnosis. However, individual differences, diseases and other factors will influence characteristics of the optic disc. Therefore, optic disc location methodology involves extensive research interest.

The existing fundus optic disc location methods can be divided into two categories. One category is based on characteristics of the optic disc itself. For example, Lalonde relied on the combination of the Hausdorff-based template matching technique and the pyramidal decomposition for large scale object tracking [2]. Walter realized optic disc location by using the Watershed Transformation [3]. These me-

*Corresponding author: Zhitao Xiao, School of Electronics and Information Engineering, Tianjin Polytechnic University, No. 399, Binshuixi Road, Xiqing District, Tianjin 300160, China. Tel.:+86-22-83955418; Fax: +22-83955164; E-mail: xiaozhitao@tjpu.edu.cn. 
thods are based on the circular structure and higher luminance of the optic disc. Another category is based on structural relation between optic disc and fundus blood vessels. For instance, Tobin used characteristics of vascular structure to extract statistical features related to vascular density, orientation and thickness to locate optic disc [4]. Hoover developed a novel algorithm named fuzzy convergence to determine the origination of blood vessel, which helps to determine the optic disc location [5]. What is more, Li realized automatic localization of the optic disc in fundus images based on 'blood vesselslike' cross-network [6]. However, optic disc is located in the convergent area of blood vessel network, which requires fundus blood vessel detection in advance and involves very high computational complexity.

On the other hand, many studies have been done upon optic disc segmentation. Osareh firstly removed the blood vessels in the optic disc using morphological preprocessing, and then extracted the optic disc with the use of the active contour model [7]. Li used morphological preprocessing method to remove blood vessels first, and then segment the optic disc automatically using an improved random walk method [8]. Chen eliminated blood vessels first using the adaptive LAB color space morphology method, and then obtained the edge of the optic disc with the use of the aided geometric active contour model according to the positioning results [9]. For all the optic disc segmentation methods mentioned above, they mainly involve two major problems. Firstly, morphological transformation is nonlinear and irreversible. As a result, the edge character of the optic disc will be changed when using globally unified and fixed structural element. Secondly, parameter active contour model requires an accurate initial edge as its inaccuracy will result in low accuracy edge detections.

There are many factors which can affect location and segmentation results, such as low contrast between the target and background, non-uniform illumination, different target sizes. These factors may cause incorrect segmentation or even target loss. Phase information, which does not change with contrast or brightness, is noise resistant and consistent with human visual system. As a result, we propose a new fundus optic disc localization and segmentation method based on phase congruency in this paper.

The following paragraphs are organized as follows. In Section 2, phase congruency model is introduced. Based on this model, detailed optic disc localization and segmentation algorithm are presented in Section 3. Finally, experiment results and conclusion are given in Section 4 and 5, respectively.

\section{Feature detection using phase congruency}

Feature detection using phase congruency makes use of phase congruency instead of using image gray information and it defines feature points as those at which phases of Fourier components are most consistent. As a result, it requires no assumption for waveform. The phase congruency function was defined by Morrone and Owens [10]:

$$
P C(x)=\max _{\bar{\phi}(x) \in[0,2 \pi]} \frac{\sum_{n} A_{n} \cos \left(\phi_{n}(x)-\bar{\phi}(x)\right)}{\sum_{n} A_{n}}
$$

where the value of $\bar{\phi}(x)$ that maximizes value of Eq. (1) is defined as the weighted mean of local phases of all Fourier terms at the point. Then PC was developed by Peter Kovesi [11]. 


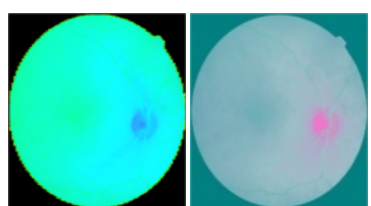

(a)

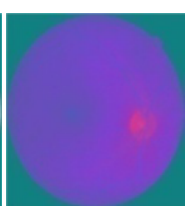

(c)

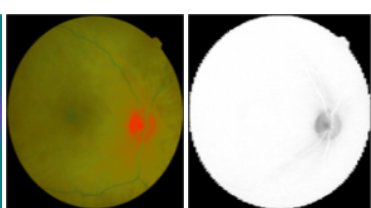

(d)



(f)

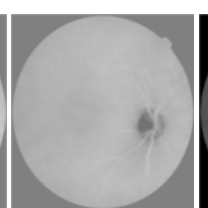

(g)

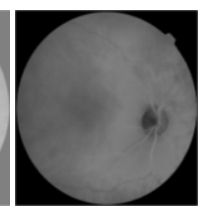

(h)

Fig. 1. Different color space and channel images. (a)-(d) Fundus images in HSV, LAB, YUV and YIQ color space. (e)-(h) Fundus images of S, A, V and I channels.

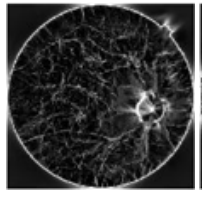

(a)

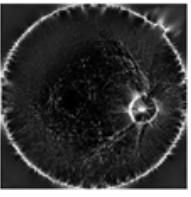

(b)

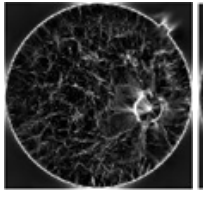

(c)

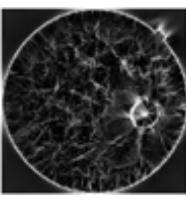

(d)



(e)

Fig. 2. Feature detection images. (a)-(d) Phase congruency images of S, A, V and I channels. (e) Result image after product of (a)-(d).

\section{Fundus optic disc localization and segmentation method based on phase congruency}

Firstly, feature detection is performed using PC model on four channel images with good contrast between the optic disc and background, respectively. Secondly, to obtain distinguishing optic disc features, multiplication operation is applied on the four PC feature detection results. Thirdly, window scanning and gray accumulating are applied to locate the optic disc. Finally, iterative OTSU automatic threshold segmentation and Hough transform are performed upon the location images, thus the final optic disc segmentation result is obtained.

\subsection{Color spaces and channels selection}

The HSV model is suitable for image processing algorithm which perceives color characteristics through human visual system. The S channel of HSV color space represents image saturation, and it is closely connected to people's way of sensing color. Optic disc has the highest contrast compared to the background in related color space and well preserved in ' $\mathrm{S}$ ', ' $\mathrm{A}$ ', ' $\mathrm{V}$ ' and ' $\mathrm{I}$ ' channels as shown in Figures 1(e)-1(h), which is conducive to locate optic disc in fundus images.

\subsection{Fundus image feature detection using phase congruency}

The implementation steps are as follows:

- Feature detection results are obtained for four channel fundus images above with the use of PC.

- Multiplication operation is applied on the four PC images to obtain final distinguishing optic disc feature detection results, as shown in Figure 2(e).

\subsection{Fundus optic disc localization}

After feature detection, the position of the optic disc can be obtained by two following steps.

- Preliminary localization: 
* Firstly, a sliding window whose size is equal to the bounding rectangle of the optic disc is defined in the feature detection images. Generally, the size of the optic disc in a standard image of healthy retina is approximately one seventh of the whole image [12];

* Next, the image is scanned with the mean values of each sliding window being calculated. As the optic disc has high contrast compared to the background in the four channels, it has obvious edge features with white pixels accumulated around in the feature detection image, as shown in Figure 2(e);

* As a result, the scanned region with the largest pixel mean value is regarded as where the optic disc locates. In a word, the algorithm gives the center coordinate of the scan window with the largest pixel mean value as optic disc.

- Precise localization:

* Firstly, the accumulated gray values of the general located window are calculated along $\mathrm{X}$ and $\mathrm{Y}$ direction and the maxima are recorded as Xmax and Ymax respectively;

* Next, the accumulated gray values are compared with two thresholds to locate optic disc precisely. The partial image in the general located window, whose accumulated gray values along $\mathrm{X}, \mathrm{Y}$ direction exceed $0.5 * \mathrm{Xmax}$ and $0.5 *$ Ymax respectively, will be reserved;

* Then, the precise localization of optic disc is obtained as the center coordinate of the reserved part in the general located window.

\subsection{Fundus optic disc segmentation}

The optic disc is segmented with the use of the location effect. Firstly, ' $A$ ' channel image of optic disc is used as the original fundus image and then a rectangular area which is slightly larger than the size of optic disc is cut out from the image according to the location result.

As for the iterative OTSU automatic threshold segmentation, it mainly involves three steps. Firstly, the initial image is separated into the background area P1 and the initial target area P2 using OTSU method. Secondly, a new threshold is obtained using OTSU method again in the original target area P2. Thirdly, the initial image is segmented based on the new threshold.

Lastly, Circular Hough Transform [13] was used to segment the circle-like optic disc.

The iterative OTSU has better stability than the OTSU method. With the use of the iterative OTSU method, most noise can be eliminated as shown in Figure 3.

\section{Experiment results and analysis}

We have tested fundus images of different qualities in clinic and STARE [14], DRIVE [15] and MESSIDOR [16] image databases. PC model with four scales and six orientations is employed

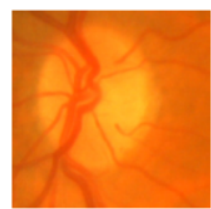

(a)

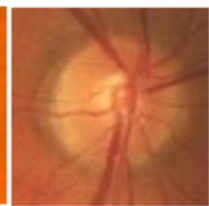

(b)

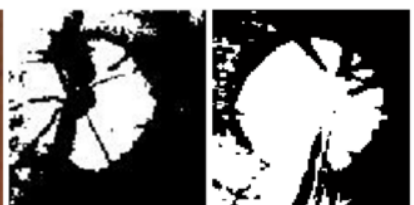

(c)

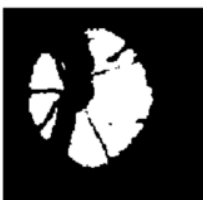

(e)

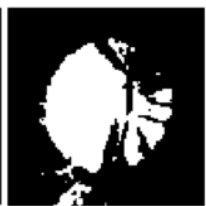

(f)

Fig. 3. Binarization results of iterative OTSU. (a), (b) Original images. (c), (d) Binary images using OTSU. (e), (f) Binary images using iterative OTSU. 
throughout the experiment. Parameters involved in iterative OTSU automatic threshold segmentation and Hough transform are selected automatically.

\subsection{Optic disc location experiment}

In order to test the stability of the method, fundus images with different brightness and contrast and seriously diseased images in STARE database are used. And the results are shown in Figure 4.

All fundus images including healthy and diseased ones in DRIVE dataset are employed for test. If the pixel location indicated by the proposed method falls within the boundaries of the optic disk, then it is considered a correct detection. Location results are shown in Table 1.

\subsection{Optic disc segmentation experiment}

For optic disc segmentation, clinic images are tested with results shown in Figure 5.

Table 1

The location success rate of proposed method and literature reviewed methods

\begin{tabular}{lllll}
\hline Dataset & total number of pictures & {$[4]$} & {$[5]$} & the proposed method \\
\hline DRIVE & 40 & $95.0 \%$ & $97.5 \%$ & $100 \%$ \\
\hline
\end{tabular}



(a)

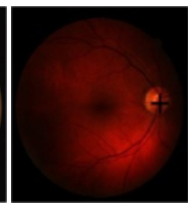

(b)

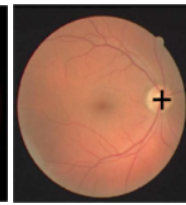

(c)

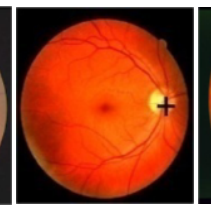

(d)



(e)



(g)

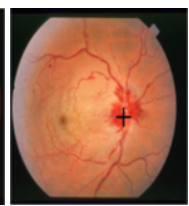

(h)

Fig. 4. Optic disc location results of fundus images. (a) Normal image. (b) Dark image. (c) Brightened image. (d) Graystretched image. (e)-(h) Seriously diseased fundus images in STARE database.



(a)

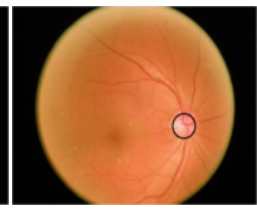

(b)

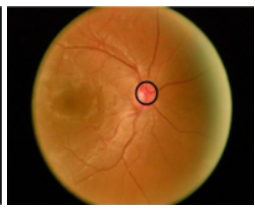

(c)

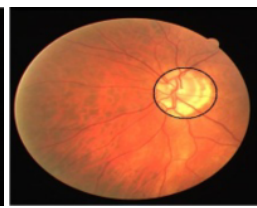

(d)

Fig. 5. Segmentation results of clinic fundus images.

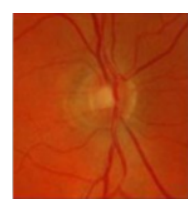

(a)

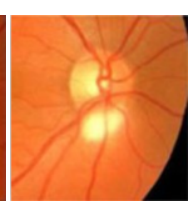

(b)

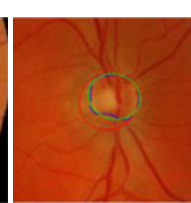

(c)

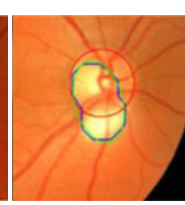

(d)

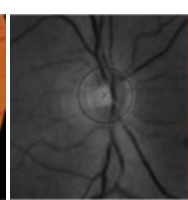

(e)

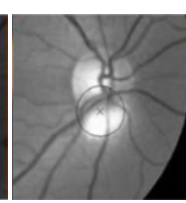

(f)

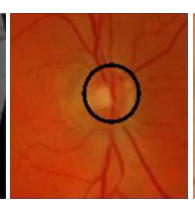

(g)

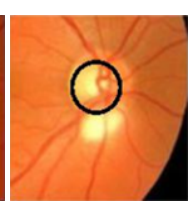

(h)

Fig. 6. Segmentation results of typical diseased images in MESSIDOR. (a), (b) Original images. (c), (d) Segmentation results of [17]. (e), (f) Segmentation results of [18]. (g), (h) Segmentation results of the proposed method. 
Table 2

The ratio of different segmentation effect images to all images in DRIVE

\begin{tabular}{lllll}
\hline Method & Excellent & Good & Fair & Bad \\
\hline Lupascu's method & $62.5 \%$ & $7.5 \%$ & $25 \%$ & $5 \%$ \\
The proposed method & $70 \%$ & $25 \%$ & $5 \%$ & $0 \%$ \\
\hline
\end{tabular}

From Figure 5, we could conclude that the proposed method can give good segmentation results for normal, mild, moderate and seriously diseased fundus images.Besides, seriously diseased images in MESSIDOR database are also employed for test. As shown in Figure 6, the proposed method has better segmentation effect than the methods developed in [17,18].

The overlap ratio $\mathrm{S}$ is calculated to further evaluate the performance of each optic disc segmentation method. $\mathrm{S}$ is given by the following equation:

$$
S=\frac{\operatorname{Area}(G T \cap C)}{\operatorname{Area}(G T \cup C)}
$$

where GT is the real optic disc area and C is the result obtained by image segmentation method. Using the overlap ratio $\mathrm{S}$, segmentation results can be evaluated and categorized from excellent to bad. The segmentation result is considered as 'Excellent' when $1 \geq \mathrm{S} \geq 0.7$, as 'Good' when $0.5 \leq \mathrm{S}<0.7$, as 'Fair' when $0<\mathrm{S}<0.5$ and as 'Bad' when the optic disc could not be found. All images in DRIVE fundus database are tested .The segmentation results are shown in Table 2.

The 'Excellent' and 'Good' classes in the proposed method account $95 \%$, which is $25 \%$ higher than that obtained using Lupascu's method. Meanwhile, the 'Fair' class reduces to $5 \%$ and the 'Bad' class is eliminated. As a result, we could conclude that the proposed method is more accurate and more robust.

\section{Conclusion}

This paper proposes a new fundus optic disc localization and segmentation method based on PC. Firstly, original images are transformed into LAB, YUV, YIQ and HSV color spaces. Secondly, four channel images with good contrast between the optic disc and the background are processed with the use of PC. Thirdly, window scanning and gray accumulating are applied to locate the optic disc. Finally, iterative OTSU automatic threshold segmentation and Circular Hough transform are performed on location images. The method has been tested upon clinic images and images in DRIVE, STARE and MESSIDOR databases. The experimental results show that the optic disc can be located and segmented accurately.

\section{Acknowledgement}

This work is supported by National Natural Science Foundation of China under grant No. 61102150, Tianjin Science and Technology Supporting Key Project of China under grant No. 13ZCZDGX02100 
and No. 14ZCZDGX00033.

\section{References}

[1] J.L. Wen, Z.Z. Yan et al., Novel lattice boltzmann method based on integrated edge and region information for medical image segmentation, Bio-Medical Materials and Engineering 8 (2013), 1247-1252.

[2] M. Lalonde, M. Beaulieu and L. Gagnon, Fast and robust optic disk detection using pyramidal decomposition and hausdorff-based template matching, IEEE Transactions on Medical Imaging 20 (2001), 1193-1200.

[3] T. Walter, J.C. Klein and P. Massin, A contribution of image processing to the diagnosis of diabetic retinopathy, detection of exudates in color fundus images of the human retina, IEEE Transactions on Medical Imaging 21 (2002), 12361243.

[4] K.W. Tobin, E. Chaum, V.P. Govindasamy et al., Detection of anatomic structures in human retinal imagery, IEEE Transaction on Medical Imaging 26 (2007), 1729-1739.

[5] A. Hoover and M. Goldbaum, Locating the optic nerve in a retinal image using the fuzzy convergence of the blood vessels, IEEE Transaction on Medical Imaging 22 (2003), 951-958.

[6] J. Li, H. Chen and X. Zhang, Automatic localization of optic nerve head in the fundus images based on cross-network, Journal of Electronics \& Information Technology 31 (2009), 1170-1174.

[7] J. Lowell, A. Hunter, D. Steel et al., Optic nerve head segmentation, IEEE Transactions on Medical Imaging 23 (2004), 256-264.

[8] J. Li, H. Chen et al., A novel segmentation method for the optic nerve head in the retinal image, Signal Processing 25 (2009), 841-846.

[9] J. Li, H. Chen et al., Automated detection of optic nerve boundary based on LAB color space, ACTA Automatica Sinica 35 (2009), 104-106.

[10] M.C. Morrone and R.A. Owens, Feature detection from local energy, Pattern Recognition Letters 6 (1987), $303-313$.

[11] P. Kovesi, Image features from phase congruency, J. Comput. Vis. Res. 1 (1999), 2-27.

[12] S. Liu and J. Chen, Detection of the optic disc on retinal fluorescein angiograms, J. Med. Biol. Eng. 31 (2011), $405-412$.

[13] R.O. Duda and P.E. Hart, Use of the hough transformation to detect lines and curves in picture, Commun. ACM 15 (1972), 11-15.

[14] A. Hoover, Structured analysis of the retina [EB/OL], http://www.ces.clemson.edu/ ahoover/stare, 2000.

[15] Image Science Institute, http://www.isi.uu.nl/Research/Databases/DRIVE, Aug. 5, 2014.

[16] Download Images Section, MESSIDOR: Digital Retinal Images, MESSIDOR TECHNO-VISION Project, France, [Online], Available: http://messidor.crihan.fr/ download-en.php.

[17] H. Yu, E.S. Barriga, C. Agruto et al., Fast localization and segmentation of the optic disk in retinal images using directional matched filtering and level sets, IEEE Transactions on Information Technology in Biomedicine 16 (2012), 644657.

[18] A. Aquino, M.E. Gegundez-arias and D. Marin, Detecting the optic disc boundary in digital fundus images using morphological, edge detection, and feature extraction techniques, IEEE Transactions on Medical Imaging 29 (2010), 18601869 . 